\title{
Patient-Specific Simulation of Carotid Artery Stenting Using Computational Fluid Dynamics
}

\author{
Juan R. Cebral ${ }^{1}$, Rainald Löhner ${ }^{1}$, Orlando Soto ${ }^{1}$, \\ Peter L. Choyke ${ }^{2}$, and Peter J. Yim ${ }^{2}$ \\ ${ }^{1}$ School of Computational Sciences, George Mason University, 4400 University Drive, \\ M.S. 4C7, Fairfax, Virginia 22030, USA \\ \{jcebral, lohner, soto\}@gmu.edu \\ ${ }^{2}$ Diagnostic Radiology Department, National Institutes of Health, Building 10, Room \\ 1C660, Bethesda, Maryland 20892, USA \\ \{pyim, pchoyke\}@nih.gov
}

\begin{abstract}
An image-based computational methodology to predict the outcome of carotid artery stenting procedures is presented. Anatomically realistic models are reconstructed from contrast-enhanced magnetic resonance angiography images using deformable models. Physiologic flow conditions are obtained from phase-contrast magnetic resonance angiography data. Finite element flow calculations are obtained before and after modifying the anatomical models in order to simulate stenting procedures. The methodology was tested on image data from a patient with carotid artery stenosis. Significant changes in the blood flow through the common carotid and internal carotid artery were found after conducting a "virtual stenting" intervention. Pending experimental validation, this methodology may potentially be used to plan and optimize vascular stenting procedures on a patient-specific basis.
\end{abstract}

\section{Introduction}

Stroke is the leading cause of long-term disability and third cause of death after cancer and heart disease in the western world. Carotid artery atherosclerosis is a major cause of stroke. However, to date there is incomplete knowledge of the atherosclerotic disease, its associated risks, and the optimal medical therapy ${ }^{1}$. Carotid artery stenting is being actively explored as a less invasive alternative to the traditional carotid artery endarterectomy ${ }^{2}$. Complications of angioplasty and stenting include cerebral embolism associated with intravascular manipulation, hemodynamic compromise during balloon inflation, vessel dissection, early restenosis or occlusion and cerebral hyperperfusion and intracranial hemorrhage ${ }^{3}$. Restenosis after angioplasty and stenting remains a major problem limiting the efficacy of the procedure. Even though the mechanisms of in-stent restenosis are not fully understood, stent implantation changes the geometry of the vessel creating new regions of decreased and increased shear stress that might be related to the observed restenosis patterns ${ }^{4,5,6}$. The planning of a stenting procedure and the prevention of complications are done based on the experience of the operator and the long-term outcomes are still not established. Image-based computational hemodynamics is 
increasingly been used to study genesis and progression of vascular disease ${ }^{7,8}$, enhance image-based diagnosis ${ }^{9}$ and plan surgical and interventional procedures ${ }^{10,11}$.

The aim of this paper is to present a computational modeling methodology to predict the outcome of carotid artery stenting procedures. The basis of the method is the integration of computational fluid dynamics (CFD) and medical imaging techniques. Patient-specific anatomical information and physiological flow conditions are obtained from magnetic resonance angiography (MRA) data. First, a realistic model of the carotid bifurcation is constructed from 3D contrast-enhanced MRA images. The geometry of this model is then modified to simulate the introduction of a vascular stent. Blood flow calculations are then conducted using finite element methods and physiologic conditions derived from phase-contrast MRA flow measurements. Finally, the blood flows before and after the introduction of the stent are compared. It is anticipated that this methodology will enable accurate predictions of blood flow patterns after performing a stenting procedure. These predictions might potentially be used to design, plan and optimize stenting interventions for individual patients ${ }^{11}$.

\section{Methods}

\subsection{Magnetic Resonance Angiography Data}

The anatomical information used to construct realistic 3D geometrical models of the carotid artery bifurcation was obtained from high-resolution 3D contrast-enhanced MRA images. These images were taken using an intra-venous injection of bolus of Gd-DTPA on a $1.5 \mathrm{~T}$ GE scanner.
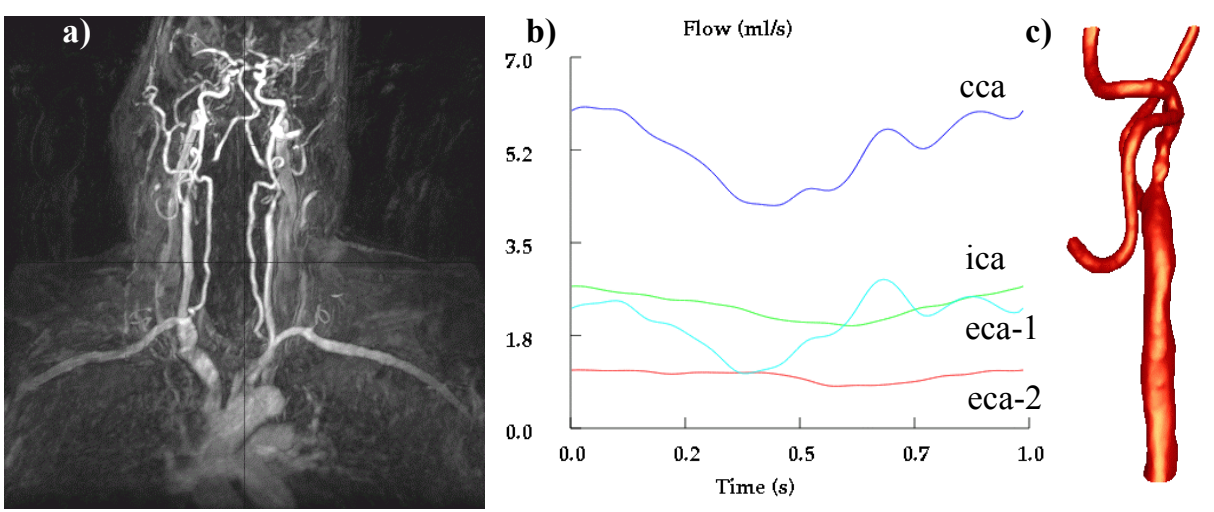

Fig. 1. MRA data: a) MIP rendering of contrast-enhanced MRA images; b) flow-rate curves obtained from phase-contrast MRA velocity measurements; c) model of the carotid bifurcation.

Blood flow velocity measurements were taken on 2D cross-sectional planes using gated cine phase-contrast MRA. Time-dependent flow curves were obtained by integration of the velocity profiles over the cross-sectional area of the carotid artery. 
Flow curves above and below the bifurcation were used to impose physiologic flow boundary conditions. The maximum intensity projection (MIP) of the anatomical images of a patient with moderate carotid artery stenosis is shown in figure 1a. The flow curves obtained in the common carotid artery (CCA), the internal carotid artery (ICA) and the external carotid artery (ECA) are presented in figure $1 \mathrm{~b}$. The final anatomical model of the carotid bifurcation is shown in figure $1 \mathrm{c}$.

\subsection{Anatomical Model Construction}

Accurate reconstruction of vessel anatomies from medical images is a challenging problem due to image artifacts, noise and limited resolution. However, many segmentation algorithms have been devised. We use a semi-automatic technique based on cylindrical deformable models ${ }^{12}$. With this approach each branch of the carotid artery is reconstructed independently. In this manner, problems associated to self-intersections or intersections with other close arteries or veins present in the images are avoided.

Once a surface triangulation was constructed for each arterial branch, a surfacemerging algorithm ${ }^{13}$ was used to obtain a watertight surface model of the carotid bifurcation. In the sequel, we refer to this model as the pre-operative model. This surface model was then used as a support surface to generate a volumetric finite element grid. Given a desired element size distribution, specified via background grids and sources, a new surface triangulation was generated using an advancing front technique ${ }^{14}$. Either linear or quadratic interpolation can be used to reposition points on the support surface. Topological constraints were used to avoid jumps between different arterial branches. The space inside the new triangulation was then filled with tetrahedral elements using a traditional advancing front grid generation method ${ }^{15}$.
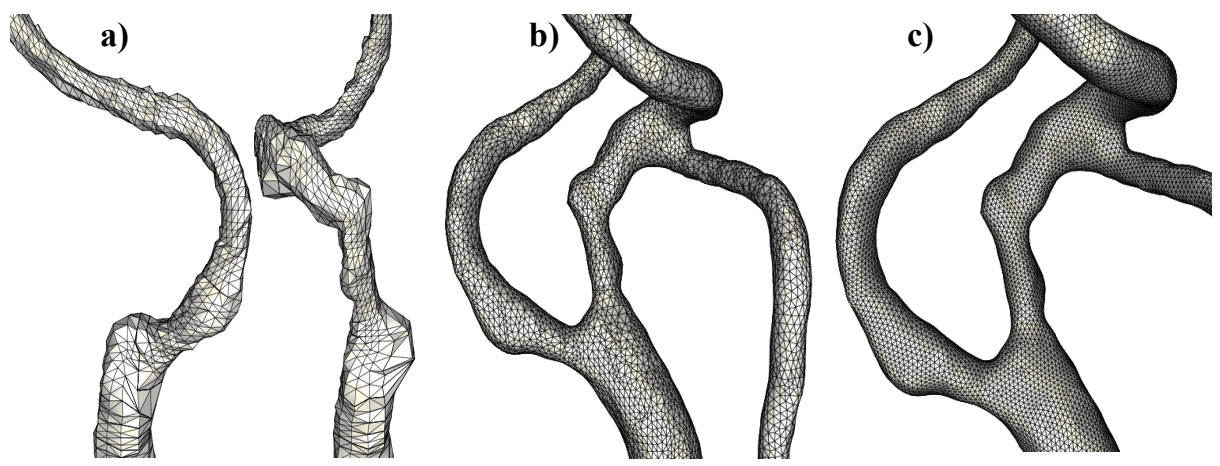

Fig. 2. Anatomical model before stenting: a) reconstructed arterial branches; b) merged model; c) surface of the finite element grid.

The element size was selected to yield approximately ten points across the smallest cross-section, resulting in roughly 0.5 million tetrahedral elements. The grid 
generation process is illustrated in figure 2. The reconstructed arterial branches and the bifurcation model after merging are shown in figures $2 \mathrm{a}$ and $2 \mathrm{~b}$, respectively.

A rigid flow-through phantom of the carotid bifurcation under steady flow conditions as well as MRA data of normal human subjects have previously been used to validate the methodology 9 . These studies show that MRA data can be used to accurately model blood flow in the carotid artery.

The surface model of the carotid bifurcation was then modified to simulate the presence of the stent. A cylindrical surface representing the stent was first created along the axis or centerline of the ICA. The arterial centerline was automatically extracted from the previous finite element grid using a skeletonization procedure operating on tetrahedral meshes, devised for visualization of blood flows in realistic arterial models ${ }^{16,17}$. The final geometrical model was then obtained by merging the pre-operative and the stent models. In what follows, we refer to this model as the post-operative model. A new finite element grid was generated using the same element size distribution as in the pre-operative case. The geometry of the stent, the post-operative model, and the corresponding finite element grid are shown in figure $3 \mathrm{a}, 3 \mathrm{~b}$ and $3 \mathrm{c}$, respectively

a)

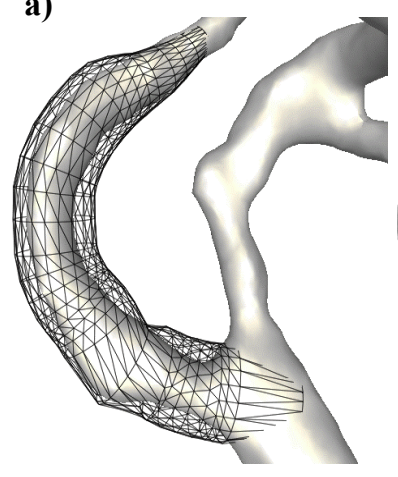

b)

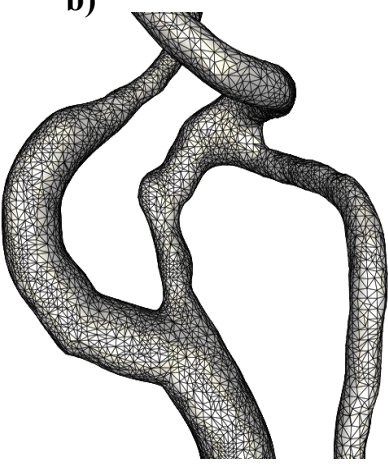

c)

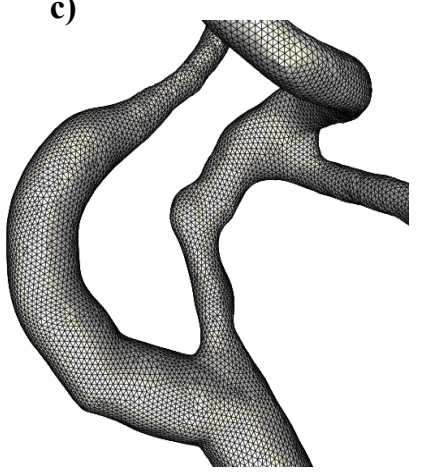

Fig. 3. Introduction of a virtual stent: a) stent geometry; b) anatomical model including after stenting; c) surface of the finite element grid.

\subsection{Blood Flow Modeling}

The time-dependent, non-linear Navier-Stokes equations for an incompressible, Newtonian fluid were used to model the blood flow ${ }^{18}$. The Newtonian approximation yields reasonable results in large arteries such as the carotids ${ }^{19}$. The vessel walls were assumed to be rigid, mainly due to a lack of pressure and elasticity information required to model compliant walls. However, it is known that atherosclerotic arteries tend to be stiffer than normal arteries; therefore this assumption is not entirely unjustified. In future studies we intend to gather all the necessary information to incorporate wall compliance, and conduct fluid-structure interaction calculations 9 . 
The fluid equations were solved using a fully implicit time discretization algorithm based on a stabilized fractional step method and linear finite element spatial discretizations ${ }^{20}$. Explicit time integration schemes, although very accurate, impose a limit on the maximum time-step size that can be used which is several orders of magnitude smaller than the period of the cardiac cycle. Thus, using such methods requires several thousands of time-steps per cardiac cycle. However, implicit schemes allow arbitrary time-step sizes, which are then selected in order to yield an accurate solution (e.g. 100 time-steps per heartbeat).

Physiologic flow boundary conditions are imposed using the flow curves derived from the phase-contrast MRA velocity measurements. At the boundaries where timedependent flows are prescribed, a velocity profile is computed as the superposition of Womersley solutions ${ }^{21}$ corresponding to different Fourier modes of the flow waveform $^{22}$.

\section{Results}

Two blood flow calculations were performed, corresponding to the pre- and postoperative models. In the pre-operative model, time-dependent flows were prescribed at the ICA and ECA's. Traction-free boundary conditions were used in the CCA, imposing a zero pressure $(p=0)$, thus the computed pressures represent pressure drops relative to the entrance of the CCA. During this simulation, the pressure drop between the CCA and the ICA were stored. For the post-operative model, time-dependent flows were prescribed at the ECA's, zero pressure was imposed at the CCA and the time-dependent pressure drop calculated in the pre-operative model was imposed at the ICA. These boundary conditions assume that the flow in the ECA's and the pressure gradient in the ICA do not change significantly after the implantation of the stent. These assumptions, which may be reasonable for cases of moderate stenosis, enable the prediction of the new flow pattern and wall shear stress distribution after stenting. However, further testing and validation of these assumptions are required.
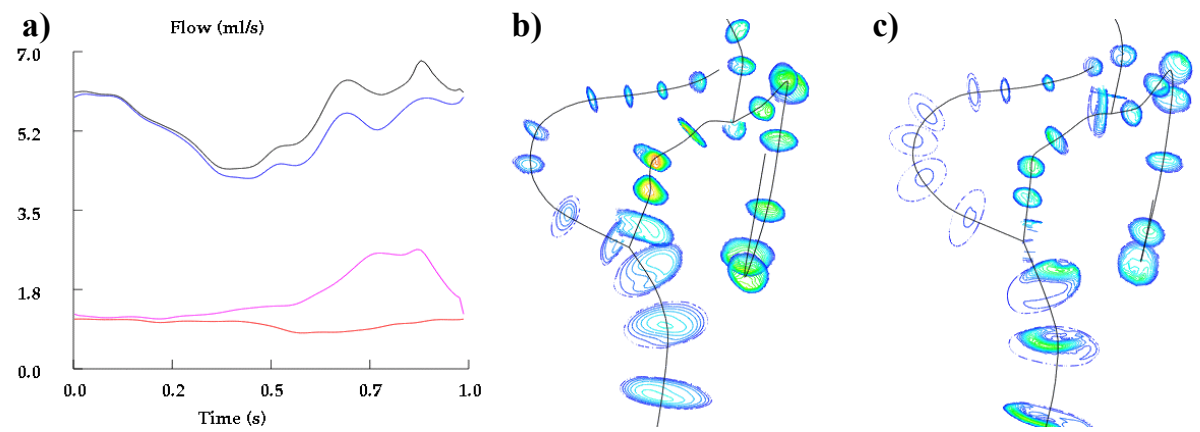

Fig. 4. a) Change in the flow rate curves through the CCA and ICA after implantation of the vascular stent; b) velocity contours before stenting; c) velocity contours after stenting. 
Both flows through the CCA and the ICA change due to the introduction of the stent in the ICA. The stent size is approximately $3.7 \mathrm{~cm}$ in length and $2.8 \mathrm{~mm}$ in radius. The change in the time-dependent flow curves precipitated by the introduction of the stent is shown in figure $4 \mathrm{a}$. Quantitatively, the flow through the CCA increases by $14 \%$ at peak systole and by $4 \%$ at end diastole. In the ICA the flow increases by $156 \%$ at peak systole and $21 \%$ at end diastole. Average flows during a cardiac cycle through the CCA and ICA change by $6 \%$ and $63 \%$, respectively. Velocity contours at peak systole before and after stenting are plotted in a series of cuts normal to the arterial axis in figures $4 b$ and $4 c$, respectively.

The change in the flow pattern and wall shear stress distribution is illustrated in figures 5 . Figures $5 \mathrm{a}$ and $5 \mathrm{~b}$ show velocity contours in a cut parallel to the arterial axis while figures $5 \mathrm{c}$ and $5 \mathrm{~d}$ show the wall shear stress maps at peak systole. It is interesting to note much stronger secondary flows after stenting in the internal and common carotids due to the increased flow.
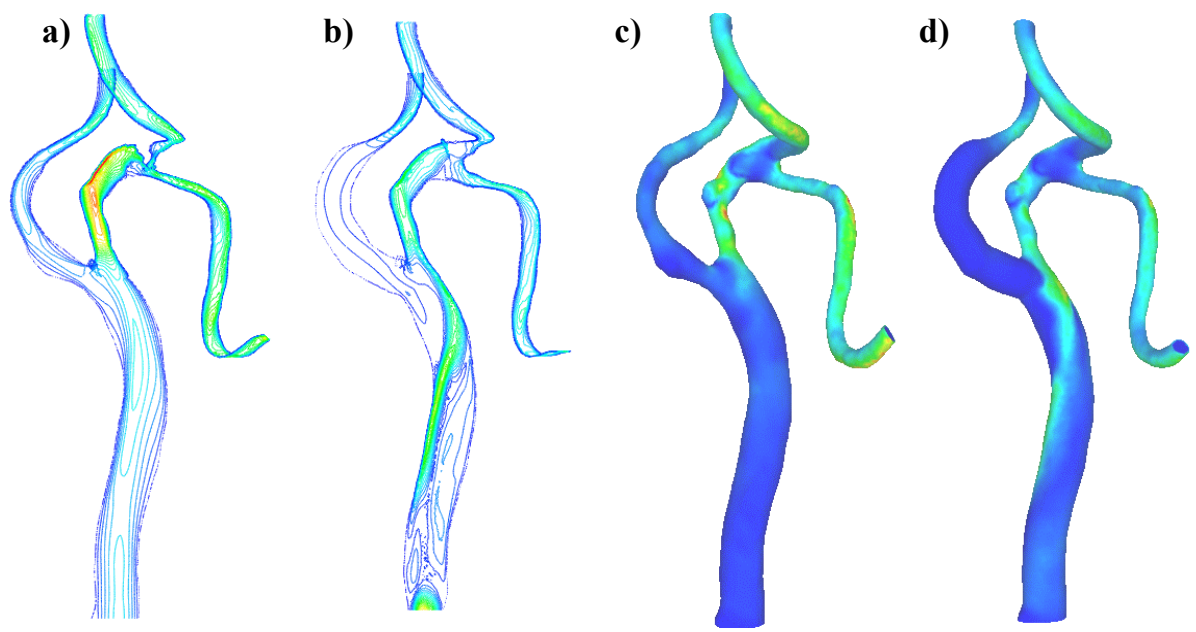

Fig. 5. Flow visualization at peak systole: velocity contours in a cut along the arterial axis before (a) and after (b) stenting; wall shear stress distributions before (c) and after (d) stenting.

Flow visualizations at end diastole and peak systole before and after the stenting procedure are shown in figure 6 . Blood flow velocity magnitudes are shown on a cut plane through the stenosis in the ICA and the CCA. Velocity profiles on a crosssectional cut through the stenosis are displayed as vectors. Secondary flows in these cross-sections are also shown. The velocity profiles become more skewed towards the stent walls.

\section{Discussion}

An image-based computational methodology to predict patient-specific blood flow in the carotid artery after a stenting procedure has been presented. Anatomical and 
physiologic data are obtained from MRA images and used to construct finite element models of the carotid artery hemodynamics.

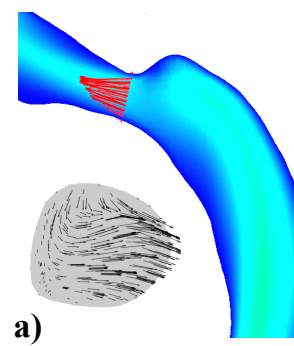

a)

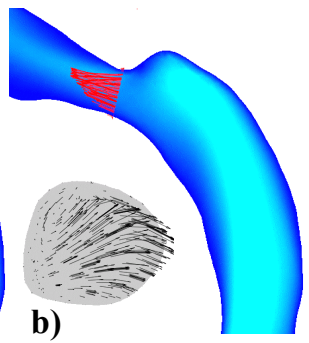

b)

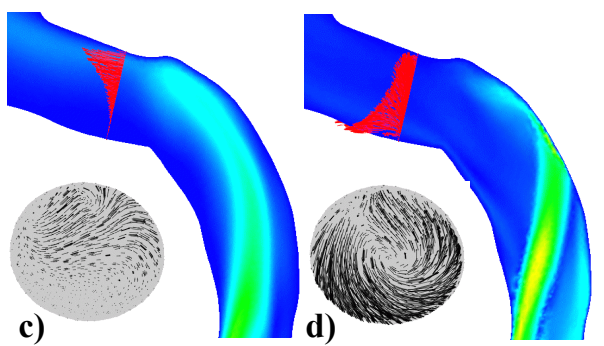

Fig. 6. Flow visualization showing velocity magnitudes, velocity profiles and secondary flows in the region of the stenosis: a) before stenting, end diastole; b) before stenting, peak systole; c) after stenting, end diastole; d) after stenting, peak systole.

Significant changes in the blood flow rates as well as average flows through both the common carotid and the internal carotid arteries were found after the introduction of a vascular stent into the internal carotid artery. The stenting procedure also changes significantly the flow pattern and the wall shear stress distribution. These predictions are particularly important in order to study complications of the stenting procedures such as restenosis, hyperperfusion syndrome and cerebral hemorrhage.

The significance of these results is that, pending experimental validation, the present methodology can be used to predict the outcome and therefore optimize carotid stenting procedures.

Limitations of the methodology include the assumption of rigid vessel walls, errors in the anatomical reconstruction and errors in the velocity measurements using MRA data. Even though the rigid wall assumption may be reasonable for stenotic arteries, wall compliance may play an important role in the development of restenosis at the ends of the stent. Future work will focus on quantification of these errors, validation studies using multi-modality image data, and verification of the post-operative boundary conditions.

\section{References}

1. Connors III, J.J.: The Nature of Cervical Carotid Stenosis. Techniques in Vascular and Interventional Radiology, 3(2) (2000) 62-64.

2. Vitek, J.J., Roubin, G.S., New, G., Al-Mubarek, N., Iyer, S.S.: Carotid Stenting. Techniques in Vascular and Interventional Radiology, 3(2) (2000) 75-85.

3. Meyers PM, Higashida RT, Phatouros C, Malek AM, Lempert TE, Dowd CF, Halbach VV: Cerebral Hyperperfusion Syndrome after Percutaneous transluminal Stenting of the Craniocervical Arteries. Neurosurgery, 47(2), (2000) 335-345.

4. Berger, S.A., Jou, L.D.: Flow in Stenotic Blood Vessels. Annual Review of Fluid Mechanics, 32 (2000) 347-82. 
5. Jou, L.D., Saloner, D.: A Numerical Study of Magnetic Resonance Angiography Images for Pulsatile Flow in the Carotid Bifurcation. Medical Engineering and Physics, 20(9) (1998) 643-52.

6. Wentzel, J.J., Whelan, D.M., van Der Giessen, W.J., van Beusekom, H.M.M., Andhyiswara, I., Serruys, P.W., Slager, C.J., Kram, R.: Coronary Stent Implantation Changes 3D Vessel Geometry and 3D Shear Stress Distribution. J. Biomech., 33 (2000) 1287-1295.

7. Milner, J.S., Moore, J.A., Rutt, B.K., Steinman, D.A.: Hemodynamics of Human Artery Bifurcations: Computational Studies with Models Reconstructed from Magnetic Resonance Imaging of Normal Subjects. J. Vasc. Surg., 27 (1998) 143-156.

8. Moore, J.A., Steinman, D.A., Holdsworth, D.W.: Accuracy of Computational Hemodynamics in Complex Arterial Geometries Reconstructed from Magnetic Resonance Imaging. Ann. Biomed. Eng., 27 (1999) 32-41.

9. Cebral, J.R., Yim, P.J., Lohner, R., Soto, O., Marcos, H., Choyke, P.L.: New Methods for Computational Fluid Dynamics Modeling of Carotid Artery from Magnetic Resonance Angiography. Proc. SPIE Medical Imaging, 4321, paper No. 22 (2001).

10. Perktold, K., Hofer, M., Karner, G., Trubel, W., Schima, H.: Computer Simulation of Vascular Fluid Dynamics and Mass Transport: Optimal Design of Arterial Bypass Anastomoses. Proc. ECCOMAS 98, 2, John Wiley \& Sons, (1998) 484-489.

11. Taylor, C.A., Draney, M.T., Ku, J.P., Parker, D., Steele, B.N., Wang, K., Zarins, C.K.: Predictive Medicine: Computational Techniques in Therapeutic Decision-Making. Computer Assisted Surgery 4 (1999) 231-247.

12. Yim, P.J., Cebral, J.R., Mullick, R., Choyke, P.L.: Vessel Surface Reconstruction with a Tubular Deformable Model. Submitted to IEEE Trans. Medical Imaging (2001).

13. Cebral, J.R., Löhner, R., Choyke, P.L., Yim, P.J.: Merging of Intersecting Triangulations for Finite Element Modeling. J. Biomech. (2001) in press.

14. Löhner, R.: Regridding Surface Triangulations. J. Comp. Phys., 126 (1996) 1-10.

15. Löhner, R.: Automatic Unstructured Grid Generators. Finite Elements in Analysis and Design, 25 (1997) 111-134.

16. Cebral, J.R., Löhner, R.: Flow Visualization On Unstructured Grids Using Geometrical Cuts, Vortex Detection and Shock Surfaces. AIAA-01-0915 (2001).

17. Cebral, J.R., Löhner, R.: Visualization of Blood Flow Computations in Realistic Anatomical Models Using Geometrical Surface Cuts. Submitted to IEEE Trans. Visualization and Computer Graphics (2001).

18. Taylor, C.A., Hughes, T.J.R., Zarins, C.K.: Finite Element Modeling of Blood Flow in Arteries. Comput. Methods Appl. Mech. Engrg. 158 (1998) 155-196.

19. Zhao, S.Z., Xu, X.Y., Hughes, A.D., Thom, S.A., Stanton, A.V., Ariff, B., Long, Q.: Blood Flow and Vessel Mechanics in a Physiologically Realistic Model of a Human Carotid Arterial Bifurcation. J. Biomech. 33 (2000) 975-984.

20. Soto, O., Löhner, R., Cebral, J.R., Codina, R.: A Time-Accurate Implicit Monolithic Finite Element Scheme for Incompressible Flow Problems. Proc. ECCOMAS CFD, Swansea, UK (2001) to appear.

21. Womersley, J.R.: Method for the Calculation of Velocity, Rate of Flow and Viscous Drag in Arteries When the Pressure gradient is Known. J. Physiol. 127 (1955) 553-563.

22. Cebral, J.R., Lohner, R., Burgess, J.E.: Computer Simulation of Cerebral Artery Clipping: Relevance to Aneurysm Neuro-Surgery Planning. Proc. ECCOMAS, Barcelona-Spain (2000). 